\title{
Spectral Karyotyping
}

National Cancer Institute

\section{Source}

National Cancer Institute. Spectral Karyotyping. NCI Thesaurus. Code C20068.

A novel karyotyping procedure which is based on the simultaneous hybridization of 24

chromosome-specific painting probes labeled with different fluorochromes or

fluorochrome combinations. The measurement of defined emission spectra by means of

interferometer-based spectral imaging allows for the definitive discernment of all human

chromosomes in different colors. (from Schrock et al., Hum Genet 1997:101:255-62) 